\title{
Fractional Brownian motors and Stochastic Resonance
}

\author{
Igor Goychul* \\ Institute of Physics, University of Augsburg, Universitätstr. 1, D-86135 Augsburg, Germany \\ Vasyl Kharchenkd \\ Institute of Physics, University of Augsburg, Universitätstr. 1, D-86135 Augsburg, Germany and \\ Institute of Applied Physics, 58 Petropavlovskaya str., 40030 Sumy, Ukraine
}

(Dated: July 13, 2021)

\begin{abstract}
We study fluctuating tilt Brownian ratchets based on fractional subdiffusion in sticky viscoelastic media characterized by a power law memory kernel. Unlike the normal diffusion case the rectification effect vanishes in the adiabatically slow modulation limit and optimizes in a driving frequency range. It is shown also that anomalous rectification effect is maximal (stochastic resonance effect) at optimal temperature and can exhibit a surprisingly good quality. Moreover, subdiffusive current can flow in the counter-intuitive direction upon a change of temperature or driving frequency. The dependence of anomalous transport on load exhibits a remarkably simple universality.
\end{abstract}

PACS numbers: 05.40.-a, 05.10.Gg, 87.16.Uv

Such diverse research fields as anomalous diffusion and transport [1-4], Brownian ratchets [5-9] and Stochastic Resonance (SR) [10] attracted much attention over last two decades with a huge research literature produced and a number of insightful reviews written which address both fundamental aspects of nonequilibrium statistical physics and various interdisciplinary applications in physics, chemistry, biology, and technology. The existing ratchet literature is restricted mostly to normal diffusion ratchets. Here, a rectification current can emerge for the particles diffusing in some periodic and unbiased on average potential due to breaking the symmetry of thermal detailed balance by an external time-dependent driving. This in turn requires to break some spatiotemporal symmetry [ 8 , 9] , for example, the spatial inversion symmetry as in Fig. 11in the case of a fluctuating tilt ratchet [6, 7] driven by harmonic force, which we consider in this work. The emergence of net directed motion in unbiased on average systems is a strongly nonequilibrium and nonlinear effect, absent e.g. within the linear response approximation, or linear Onsager regime of nonequilibrium thermodynamics. A characteristic feature of any true ratchet is its ability to sustain a load, i.e. a force directed against the transport direction. The presence of a non-zero stopping force allows to distinguish the genuine ratchets or Brownian motors capable to do useful work from the futile ones or pseudo-ratchets [8, 9]. In any isothermal Brownian motion which never ceases the dissipative loss of energy is compensated at thermal equilibrium by the energy (heat) gain due the thermal noise of environment so that on average the classical Brownian particle has kinetic energy $k_{B} T / 2$ per degree of freedom. This ensures the absence of a net directed motion and of the total heat exchange between the particle and its environment. The directed Brownian motion requires an external source of energy - a part of it will be put into the directed motion and a part dissipated as an excess heat to the environ-

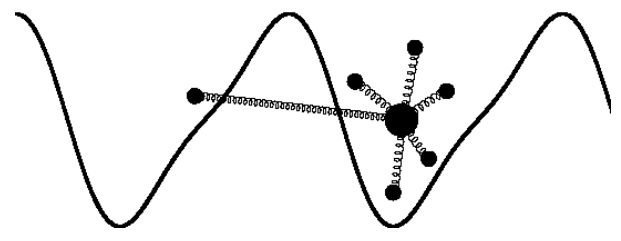

FIG. 1. Ratchet potential and central Brownian particle coupled to auxiliary Brownian particles modeling viscoelastic environment.

ment. The thermal noise plays in fact a constructive role here. First, as a sort of "lubricant" to smooth the friction and also providing thermal energy fluctuations allowing to overcome potential barriers met on the particle's pathway. Restricting to the classical world, without noise the Brownian particle would remains localized in a potential well, starting there having subthreshold energy and for a weak external driving. Therefore, in such a setup one generally expects that the rectification current response to a subthreshold driving will increase with the noise intensity. However, for a very strong noise the potential barriers cease to matter and one expects that rectification effect due to a spatial asymmetry of potential will vanish. Therefore, there should exist an optimal thermal noise intensity, or temperature which typies Stochastic Resonance (SR) in broad sense. In the focus of this Letter are the ratchet and Stochastic Resonance effects in subdiffusive transport, i.e. mean displacement grows sublinearly, $\langle\delta x(t)\rangle \propto t^{\alpha}$, with $0<\alpha<1$, in a viscoelastic environment.

The very existence of subdiffusive ratchet transport is not obvious and can be questioned. For example, within a continuos time random walk (CTRW) mechanism of subdiffusion featured by divergent mean residence times (MRTs) [1, 3] the current response to external periodic driving is asymptotically zero [11]. This clearly prohibits any asymptotic rectification effect for such fluctuating tilt 
ratchets. However, a subdiffusive rocked ratchet based on the fractional Brownian motion (fBm) [12] does exist [13], and we shall explain its unusual properties, showning in particular that the resonance nonadiabatic character of this anomalous ratchet effect is of the SR origin, and it is a genuine ratchet effect.

Theory. The Fractional Brownian motion can emerge as solution of the Generalized Langevin Equation (GLE) [14 16] for a Brownian particle with mass $m$,

$$
m \ddot{x}+\int_{0}^{t} \eta\left(t-t^{\prime}\right) \dot{x}\left(t^{\prime}\right) d t^{\prime}=f(x, t)+\xi(t),
$$

where $f(x, t)=-\partial V(x, t) / \partial x$ is a deterministic force, $\xi(t)$ is zero-mean and Gaussian-distributed thermal noise and $\eta(t)$ is the frictional memory kernel related to noise by the fluctuation-dissipation relation (FDR)

$$
\left\langle\xi\left(t^{\prime}\right) \xi(t)\right\rangle=k_{B} T \eta\left(\left|t-t^{\prime}\right|\right) .
$$

The $\mathrm{fBm}$ emerges in the overdamped limit, $m \rightarrow 0$, of a force-free motion, $f \rightarrow 0$, for a power-law frictional kernel $\eta(t)=\eta_{\alpha} t^{-\alpha} / \Gamma(1-\alpha)$, with $0<\alpha<1[\Gamma(x)$ is standard gamma-function], and the FDR-related noise $\xi(t)$, which is termed the fractional Gaussian noise (fGN) 12]. The corresponding GLE is also termed the fractional Langevin equation (FLE) 16 18]. The GLE can be derived from a standard Hamiltonian model of the Brownian motion based on the coupling of Brownian particle to a thermal bath of harmonic oscillators modeling the environment [15]. The FLE model corresponds to sub-Ohmic thermal bath characterized by the spectral bath density $J(\omega) \propto \eta_{\alpha} \omega^{\alpha}[19]$. Such a modeling requires a dense spectrum of the thermal bath, or quasi-infinite number of oscillators. Alternatively, one can model the environment by a finite set of co-moving auxiliary Brownian particles, cf. Fig. 1, with masses $m_{i}$ coupled elastically with constants $k_{i}$ to the central Brownian particle and experiencing the viscous Stokes friction (with frictional constants $\left.\eta_{i}\right)$ and uncorrelated, $\left\langle\xi_{i}(t) \xi_{j}\left(t^{\prime}\right)\right\rangle=\delta_{i j} \delta\left(t-t^{\prime}\right)$, white-noise thermal Gaussian forces $\sqrt{2 \eta_{i} k_{B} T} \xi_{i}(t)$ [20] . Considering the overdamped limit for auxiliary particles, $m_{i} \rightarrow 0$, this yields upon introduction of fluctuating viscoelastic forces, $u_{i}=-k_{i}\left(x-x_{i}\right)$ [20]:

$$
\begin{aligned}
m \ddot{x} & =f(x, t)+\sum_{i=1}^{N} u_{i}(t), \\
\dot{u}_{i} & =-k_{i} v-\nu_{i} u_{i}+\sqrt{2 \nu_{i} k_{i} k_{B} T} \xi_{i}(t),
\end{aligned}
$$

where $\nu_{i}=k_{i} / \eta_{i}$ are the relaxation rates of viscoelastic forces. The last equation for $u_{i}$ has the form of relaxation equation for elastic force introduced by Maxwell in his macroscopic theory of viscoelasticity [21] which is augmented by the corresponding Langevin force in accordance with FDR. Such a description was introduced in Refs. [13, 22] to model anomalous Brownian motion in complex viscoelastic media within a generalized
Maxwell model. Indeed, by choosing initial $u_{i}(0)$ as independent zero-mean Gaussian variables with variance $\left\langle u_{i}^{2}(0)\right\rangle=k_{i} k_{B} T$ and excluding the dynamics of auxiliary variables $u_{i}$, the GLE (11), (2) follows immediately with the memory kernel $\eta(t)=\sum_{i=1}^{N} k_{i} e^{-\nu_{i} t}$. For $N=1$, an earlier Markovian embedding of GLE with exponentially decaying memory kernel [23] is readily reproduced. Furthermore, by choosing $\nu_{i}=\nu_{0} / b^{i-1}, \quad k_{i}=$ $C_{\alpha}(b) \eta_{\alpha} \nu_{i}^{\alpha} / \Gamma(1-\alpha)$, where $b$ is a scaling parameter and $C_{\alpha}(b)$ is a fitting dimensionless constant, the above power-law kernel $\eta(t)$ can be well approximated over about $r=N \log _{10} b-2$ temporal decades between two time cutoffs, $\tau_{l}=\nu_{0}^{-1}<t<\tau_{h}=\tau_{l} b^{N-1}$. Similar scaling and approximation are well known in the anomalous relaxation theory [1, 24]. Physically, $\nu_{0}$ corresponds to a high-frequency cutoff in $J(\omega)$, or the largest medium's frequency, and $\nu_{0} / b^{N-1}$ to the slowest medium's mode, which are always present in reality. For the case $\alpha=0.5$ studied numerically in this work, the choice $b=10$, $C_{0.5}=1.3, N=12$, and $\nu_{0}=100$ provides an excellent approximation to the FLE dynamics over at least ten time decades, until $t_{\max }=10^{8}$. This is checked 13, 22, 25] by comparison with the exact solution for the position variance obtained within and GLE and FLE [14, 17] in the force-free case. We scale time in units of the (anomalous) velocity relaxation constant $\tau_{v}=\left(m / \eta_{\alpha}\right)^{1 /(2-\alpha)}$. It is assumed to be temperatureindependent in accordance with the Hamiltonian model [15, 19]. This is a standard assumption done also in other toy ratchet models $[8,9]$.

Stochastic dynamics is studied in a driven ratchet potential, $V(x, t)=U(x)-A x \cos (\Omega t)+f_{0} x$, where [7]

$$
U(x)=-U_{0}[\sin (2 \pi x / L)+0.25 \sin (4 \pi x / L)]
$$

is a spatially asymmetric periodic potential with amplitude $U_{0}$ and period $L, A$ and $\Omega$ are the amplitude and frequency of the periodic forcing, and $f_{0}$ is a load. The distance is scaled in the units of $L$, the energy in units of $\tilde{E}=m L^{2} / \tau_{v}^{2}$, force in $\tilde{F}=\tilde{E} / L$ and temperature in $\tilde{T}=\tilde{E} / k_{B}$. The role of the inertial effects can be characterized by the dimensionless parameter $r_{v}=1 /\left(\omega_{b} \tau_{v}\right)$, where $\omega_{b}=(2 \pi / L)\left(3^{3 / 8} / 2^{1 / 4}\right) \sqrt{U_{0} / m}$ is the bottom and (imaginary) top frequency of the considered potential for $A=0, f_{0}=0$. The inertial effects can only be negligible for $r_{v} \ll 1$ and not too small $\alpha$ [18]. The corresponding borderline value of $U_{0}$ corresponding to $r_{v}=1$ is in the dimensionless units $U_{0}^{*} \approx 0.0157$. For the simulations done in this work the inertial effects are very essential. This might seem paradoxical since in the Markovian approximation $\dot{x}\left(t^{\prime}\right) \rightarrow \dot{x}(t)$ to Eq. (1) the effective Markovian friction $\eta_{\text {eff }}(t)=\int_{0}^{t} \eta\left(t^{\prime}\right) d t^{\prime} \propto \eta_{\alpha} t^{1-\alpha}$ increases to infinity in the course of time. Such a Markovian approximation is, however, not affordable for the considered viscoelastic dynamics. Fig. 11illustrates this point: A part of auxiliary particles is strongly coupled to the Brownian particle. They are co-moving being strongly corre- 


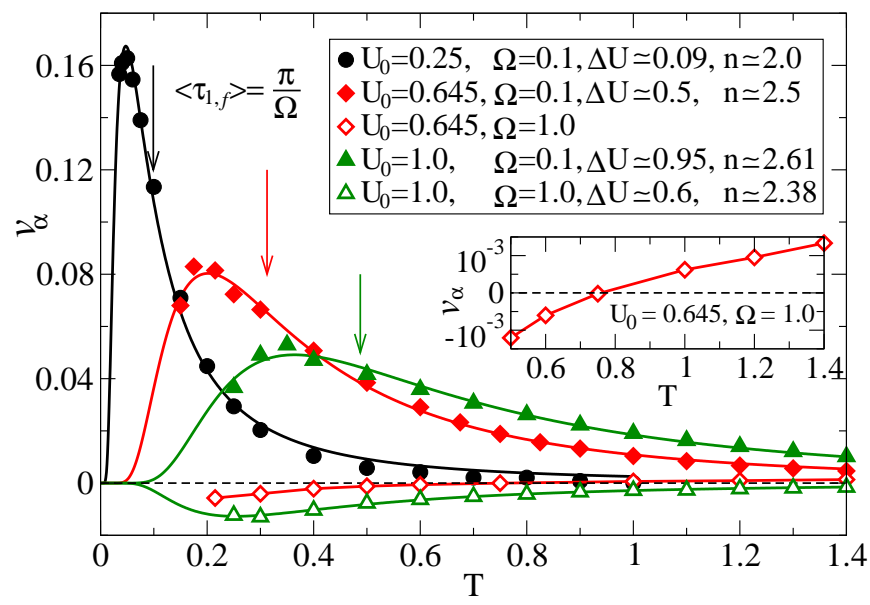

FIG. 2. (Color online) Anomalous current (subvelocity $v_{\alpha}$ ) as a function of temperature for different values $U_{0}$ and $\Omega$.

lated, reminding a polaron-like picture for quantum particles in polar media. However, weaker coupled, stronger damped, and much slower particles cannot follow immediately. They create an elastic force pulling the central particle back and retarding its overall motion. This prohibits any Markovian approximation on the level of the reduced $(x, v)$ dynamics as the corresponding slow hidden dynamics cannot be adiabatically eliminated. And nevertheless, a highly dimensional Markovian approximation with $N$ extra dimensions for overdamped auxiliary particles works remarkably well. Of course, given a finite $N$ the trully asymptotical dynamics becomes normal for $t \gg t_{\max }=b^{N-1} / \nu_{0}$. However, $t_{\max }$ grows exponentially fast with $N$ and therefore it can be totally irrelevant, as in our simulations, to figure out the correct asymptotic FLE dynamics. We take a physical limit of very large $t$ to study subdiffusive dynamics with $t_{\max }$ regarded as infinite on this time scale and define the subvelocity as $v_{\alpha}=\Gamma(1+\alpha) \lim _{t \rightarrow \infty} \frac{\langle x(t)\rangle}{t^{\alpha}}$. Practically, the corresponding values of $v_{\alpha}$ were calculated by fitting the dependence $\langle x(t)\rangle$ with the $a t^{\alpha}$ dependence, extracting the corresponding $a$ within a last time window of simulations done until $t_{\mathrm{sim}}=10^{6} \ll t_{\max }$. In all simulations we have used $n=10^{4}$ trajectories for ensemble averaging. The stochastic Heun algorithm has been implemented with double precision on a graphical processor unit (GPU) [26] which allowed to parallelize and accelerate stochastic simulations by a factor of about 50 as compare with standard CPU computing. The amplitude of the external driving was fixed at $A=0.8$, which is beyond the lowest second order of nonlinear response in $A / T$ that yields a rectification subtransport response, while the temperature, potential amplitude, and the driving frequency were varying.

Results. First, we fixed the temperature at $T=0.25$ and varied the driving frequency and the potential amplitude, cf. 20], Fig. S1. Remarkably, the rectification response is absent in the adiabatic limit $\Omega \rightarrow 0$ as in 13]. This is in a sharp contrast with the case of normal diffusion where the adiabatic current is maximal [8, [9]. Indeed, the subdiffusion and subtransport in periodic potentials turn out to be asymptotically insensitive to the potential amplitude $U_{0}$ [22]. This surprising feature is due to the influence of slugish dynamics of the medium's degrees of freedom which cannot immediately follow to a faster moving Brownian particle. They cause ultraslow dynamics on the time scale which largely exceeds the mean time spent in a potential well. In this respect, the medium's dynamics is not influenced directly by an external force. Therefore, for an adiabatically slow driving a periodic potential does not play any role in the long time limit and the ratchet transport is absent. For a very fast driving, the transport is also obviously increasingly suppressed upon increasing the driving frequency. Therefore, there should exist an optimal value of driving frequency when the corresponsing subvelocity attains a maximum, $v_{\alpha, \max }=\max _{\Omega} v_{\alpha}(\Omega)$, which is a Stochastic Resonance related effect [20]. This maximal value is optimized also with the potential amplitude. For example, $v_{\alpha, \max }$ is larger for $U_{0}=0.645$, than for $U_{0}=0.5$ and $U_{0}=1.0$, see in [20], Fig. S1 and the corresponding discussion therein. Noteworthy is also the inversion of the current direction for sufficiently large $U_{0}$ and $\Omega$. The subtransport then occurs in the conterintuitive direction, contrary to the direction predicted by the slow adiabatic tilt argumentation. A similar inversion for high-frequency driving occurs also in the normal diffusion case [7, 8].

SR-related phenomenon occurs also in the dependence of subcurrent on temperature, cf. Fig. 2, An adiabatic driving argumentation predicts $v_{\alpha}(T) \propto$ $T^{-n} \exp (-\Delta U / T)$ with $n=2$ and $\Delta U=2 U_{0}$ in the lowest order of perturbation theory over $A / T$ (quadratic response), for a weak driving, $A L \ll 2 \pi U_{0}$. This is a typical SR dependence yielding the maximum versus temperature at $T_{\max }=\Delta U / n$. We are dealing but with a strong nonadiabatic driving and hence use $\Delta U$ and $n$ in Fig. 2 as some fitting parameters. The SR origin of the maxima for sufficiently small $\Omega$ is substantiated by matching the forward tilt half-periods with the numerical mean times $\left\langle\tau_{1, f}\right\rangle$ of the jumps into the forward directions, i.e. $\left\langle\tau_{1, f}\right\rangle=\pi / \Omega$ [10], with temperature near to the transport optimization as indicated by arrows in Fig. 2. The negative current for a larger $\Omega$ is also optimized with temperature, which does not have but any relation to SR or synchronization. Morever, an inversion of the subcurrent direction with temperature is detected in Fig. 2 (see also the insert therein) for $U_{0}=0.645$ and a high-frequency driving $\Omega=1.0$. Similar temperature inversions occur also in the normal diffusion case [7].

Furthermore, the viscoelastic subdiffusion in periodic potentials is asymptotically also not affected by the potential height 22 even though a periodic driving can slightly affect it. Asymptotically, in the considered units 


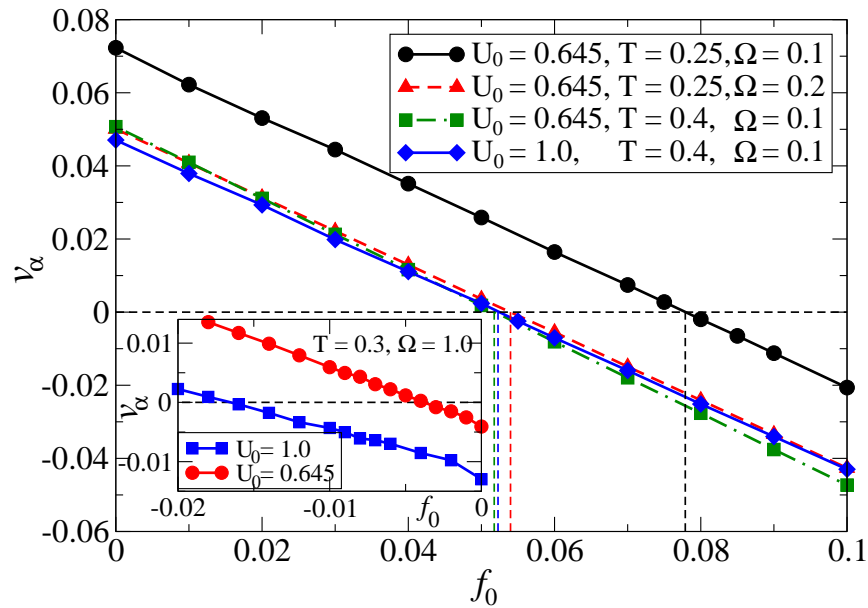

FIG. 3. (Color online) Anomalous current (subvelocity $v_{\alpha}$ ) as a function of positive load $f_{0}$ at different $U_{0}, T$ and a small driving frequency $\Omega=0.1$. Insertion shows dependencies $v_{\alpha}$ versus negative load $f_{0}$ at $T=0.3$ and some high $\Omega=1.0$ at different $U_{0}$.

$\left\langle\delta x^{2}(t)\right\rangle \sim 2 D_{\alpha} t^{\alpha} / \Gamma(1+\alpha)$ with subdiffusion coefficient $D_{\alpha}=T$. A periodic driving does affect $D_{\alpha}$. However, it is not changed strongly [13]. Therefore, the generalized Peclet number 13] $\mathrm{Pe}_{\alpha}:=v_{\alpha} L / D_{\alpha}$, which measures the coherence and quality of transport [27], can be appreciable. With lowering the barrier height in Fig. 2 the maximum of subvelocity is shifted towards smaller temperatures and the corresponding $\mathrm{Pe}_{\alpha}$ can exceed the value of one signifying thereby that a high-quality subdiffusive ratchet transport is possible.

Finally, the dependence of the directed subtransport on the load $f_{0}$ in the opposite direction is shown in Fig. 3. The existence of the stopping force shows clearly that we are dealing with a genuine ratchet effect. Given the asymptotic independence of the viscoelastic subtransport on the potential amplitude $U_{0}$ in washboard potentials 22] one expects a very simple dependence $v_{\alpha}\left(f_{0}\right)=v_{\alpha}(0)-f_{0}$ (in dimesionless units) to hold for small driving frequencies $\Omega$. Indeed, the numerical results are consistent (taking numerical errors into account) with this prediction. However, for larger $\Omega$ and the inverted transport the deviations become more pronounced.

In conclusion, with work we put forward a toy model for viscoelastic subdiffusive ratchet transport and showed that such a transport can be optimized by ambient thermal noise and frequency of the external driving due to Stochastic Resonance effects and can exhibit a surprisingly good quality.

Acknowledgment. Support of this research by the Deutsche Forschungsgemeinschaft, Grant GO 2052/1-1 is gratefully acknowledged.
* igor.goychuk@physik.uni-augsburg.de

$\dagger$ vasyl.kharchenko@physik.uni-augsburg.de

[1] B. D. Hughes, Random walks and Random Environments, Vols. 1,2 (Clarendon Press, Oxford, 1995).

[2] J.-P. Bouchaud and A. Georges, Phys. Rep. 195, 127 (1990).

[3] R. Metzler and J. Klafter, Phys. Rep. 339, 1 (2000).

[4] Fractional Dynamics: Recent Advances, eds. J. Klafter, S. C. Lim, R. Metzler (World Scientific, Singapore, 2011).

[5] A. Ajdari and J. Prost, CR Acad. Sci. Paris, Ser. II 315, 1635 (1992); R. D. Astumian and M. Bier, Phys. Rev. Lett. 72, 1766 (1994).

[6] M. O. Magnasco, Phys. Rev. Lett. 71, 1477 (1993); C. R. Doering, W. Horsthemke, and J. Riordan, 72, 2984 (1994).

[7] R. Bartussek, P. Hänggi, and J. G. Kissner, Europhys. Lett. 28, 459 (1994).

[8] P. Reimann, Phys. Rep. 361, 57 (2002).

[9] P. Hänggi and F. Marchesoni, Rev. Mod. Phys. 81, 387 (2009).

[10] L. Gammaitoni, P. Hänggi, P. Jung, and F. Marchesoni, Rev. Mod. Phys. 70, 223 (1998).

[11] I. M. Sokolov and J. Klafter, Phys. Rev. Lett. 97, 140602 (2006); E. Heinsalu, M. Patriarca, I. Goychuk, and P. Hänggi, Phys. Rev. Lett. 99, 120602 (2007); E. Heinsalu, M. Patriarca, I. Goychuk, and P. Hänggi, Phys. Rev. E 79, 041137 (2009).

[12] B. B. Mandelbrot and J. W. van Ness, SIAM Rev. 10, 422 (1968).

[13] I. Goychuk, Chem. Phys. 375, 450 (2010).

[14] R. Kubo, Rep. Prog. Phys. 29, 255 (1966).

[15] R. Zwanzig, J. Stat. Phys. 9, 215(1973); R. Zwanzig, Nonequilibrium Statistical Mechanics (Oxford University Press, Oxford, 2001).

[16] W. T. Coffey, Y. P. Kalmykov, J. T. Waldron, The Langevin Equation, 2nd ed. (World Scientific, Singapore, 2004).

[17] E. Lutz, Phys. Rev. E 64, 051106 (2001); I. Goychuk and P. Hänggi, Phys. Rev. Lett. 99, 200601 (2007).

[18] S. Burov and E. Barkai, Phys. Rev. Lett. 100, 070601 (2008).

[19] U. Weiss, Quantum Dissipative Systems, 2nd ed. (World Scientific, Singapore, 1999).

[20] See complementary material at: http://link.aps.org/supplemental/... .

[21] J. C. Maxwell, Phil. Trans. R. Soc. Lond. 157, 49 (1867).

[22] I. Goychuk, Phys. Rev. E 80, 046125 (2009); I. Goychuk and P. Hänggi, in: [4], Ch. 13, pp. 307-329; I. Goychuk, Adv. Chem. Phys. 150 (2012, in press).

[23] F. Marchesoni and P. Grigolini, J. Chem. Phys. 78, 6287 (1983); J.E. Straub, M. Borkovec, and B. J. Berne, J. Chem. Phys. 84, 1788 (1986).

[24] R. G. Palmer, D. L. Stein, E. Abrahams, P. W. Anderson, Phys. Rev. Lett. 53, 958 (1984).

[25] P. Siegle, I. Goychuk, and P. Hänggi, Europhys. Lett. 93, 20002 (2011)

[26] See on: http://www.nvidia.com/object/ cuda_home_new.html

[27] B. Lindner, M. Kostur, and L. Schimansky-Geier, Fluct. Noise Lett. 1, R25 (2001). 


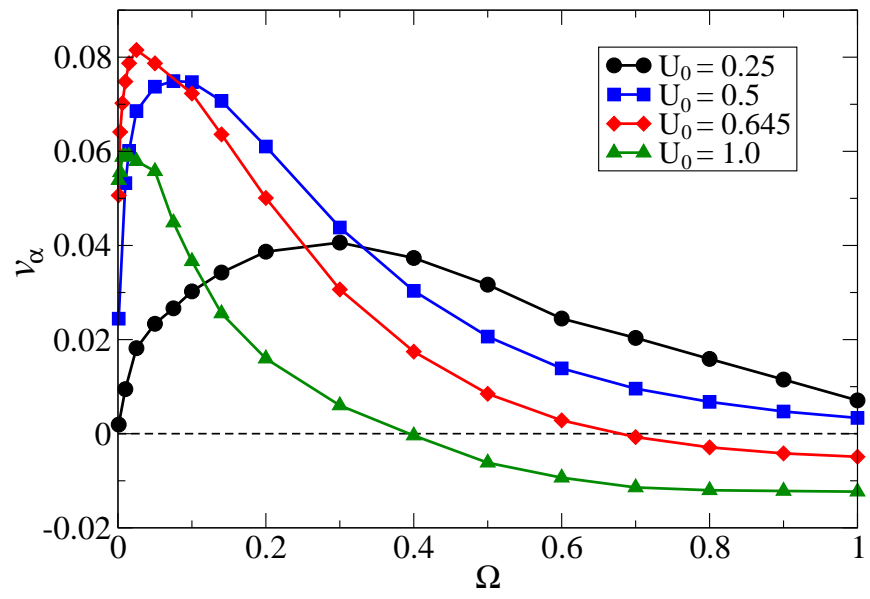

Figure S1: Anomalous current (subvelocity $v_{\alpha}$ ) as a function of the driving frequency for different $U_{0}$ at $T=0.25$ for zero load $f_{0}=0$.

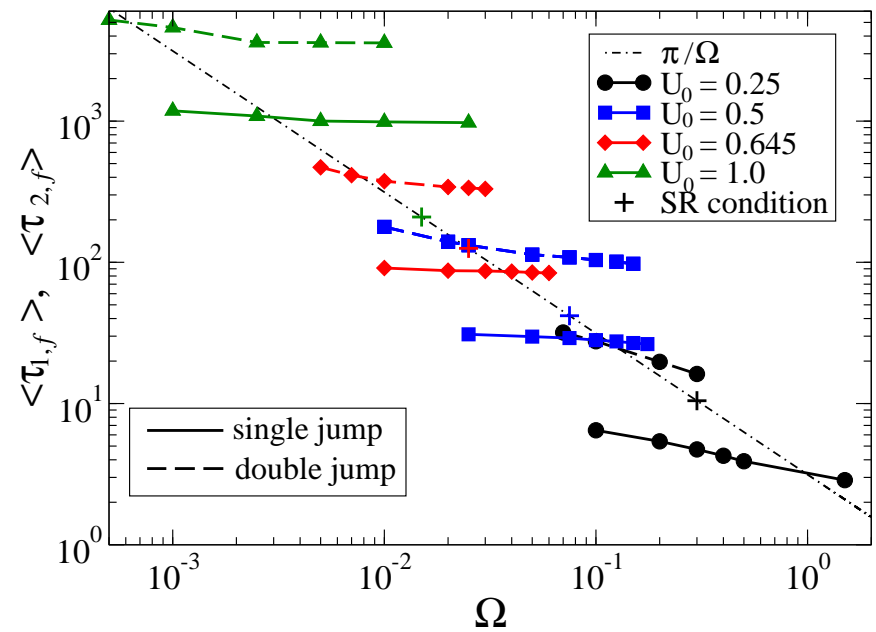

Figure S2: Mean times of transitions, $\left\langle\tau_{1, f}\right\rangle$ and $\left\langle\tau_{2, f}\right\rangle$, as functions of the driving frequency $\Omega$. The values $\Omega_{\max }$ and $v_{\alpha}\left(\Omega_{\max }\right)$ are indicated by crosses.

\section{Supplementary Material}

1. Stochastic equations of motion for a central particle surrounded by a cloud of elastically bounded Brownian particles read

$$
\begin{aligned}
m \ddot{x} & =f(x, t)-\sum_{i=1}^{N} k_{i}\left(x-x_{i}\right), \\
m_{i} \ddot{x}_{i} & =k_{i}\left(x-x_{i}\right)-\eta_{i} \dot{x}_{i}+\sqrt{2 \eta_{i} k_{B} T} \xi_{i}(t) .
\end{aligned}
$$

This equations are similar to the starting equations of motion in the purely dynamical model leading to the GLE description. The last one can be obtained by setting $\eta_{i} \rightarrow 0$, i.e. considering a purely dynamical evolution with a tremendously large number of oscillators $N \rightarrow \infty$ with a dense spectrum $\omega_{i}=\sqrt{k_{i} / m_{i}}$. The only nondynamical assymption in the dynamical model is that initially the thermal bath oscillators are canonically distributed at the temperature $T$ - like in a standard molecular dynamics setup. We replace them by a finite set of auxiliary Brownian particles.

2. Fig. S1 displays the dependence of subvelocity on external driving frequency $\Omega$ for different potential amplitudes $U_{0}$ and the fixed driving amplitude and temperature. In order to clarify the physical origin of maximal $v_{\alpha, \max }$ we have calculated the frequency-dependent mean times of the transitions to the neighboring potential well, $\left\langle\tau_{1, f}\right\rangle$, and to the second next potential well, $\left\langle\tau_{2, f}\right\rangle$. If the maximum of $v_{\alpha}$ is due to synchronization, then the transport should become optimal when the particles advance in the trasport direction over one or two potential periods during the driving half-period $\mathcal{T}_{1 / 2}=\pi / \Omega$. This means that the condition $\left\langle\tau_{1, f}\right\rangle<\mathcal{T}_{1 / 2}<\left\langle\tau_{2, f}\right\rangle$ should be obeyed. Indeed, the numerical results displayed in Fig. $\mathrm{S} 2$ show that this is the case for not too high $U_{0}$. However, since $\left\langle\tau_{1, f}\right\rangle$ and $\left\langle\tau_{2, f}\right\rangle$ are only weakly frequencydependent and thus no frequency entraintment occurs, we are dealing with the phenomenon of Stochastic Resonance rather than stochastic synchronization. Here, the response maximizes when an external driving frequency fits into an intrinsic frequency of stochastic dynamics. 